\title{
UNA PERSPECTIVA CURRICULAR DEL ENFOQUE DE LOS SISTEMAS SIMBÓLICOS EN LA EDUCACIÓN COSTARRICENSE
}

\author{
William Delgado Montoya \\ Profesor en ejercicio en la escuela \\ Lider Ricardo Jiménez de Cartago
}

Recibido 30-VIII-2004

Aceptado 7-IX-2004

\begin{abstract}
Resumen: Este artículo propone un acercamiento a la educación desde la perspectiva de la teoría de las inteligencias múltiples y la inteligencia emocional. Entendida inteligencia como la capacidad para resolver problemas, o para elaborar productos que son de valor para un determinado contexto comunitario o cultural. Por lo tanto, el éxito o fracaso escolar se podrá predecir, dependiendo de la capacidad que demuestre el niño para resolver diferentes situaciones problemáticas y la adaptación u ajuste entre un ser humano activo en crecimiento y los escenarios naturales y, sociales. Es decir, plantea la educación como una estructura social que favorecen los mecanismos de acomodación al explicar, predecir, interpretar, transmitir la cultura y la práctica social, configurando los escenarios donde se efectúan los procesos de enseñanza aprendizaje, de esta manera, el aula escolar se transforma en un espacio de interacciones entre los individuos (profesores y alumnos). En consecuencia, el enfoque simbólico en la educación es una oportunidad para realizar investigaciones que valoren la diversidad cultural, el contexto de aprendizaje de los alumnos, el estímulo de las inteligencias de cada individuo, las interacciones humanas que constantemente se modifican, para generar una búsqueda de soluciones a los problemas de una manera altruista.
\end{abstract}

Palabras clave: Currículo, inteligencia, enfoque simbólico, diversidad, inteligencias múltiples y emocional, aula escolar, contexto, educación.
"El niño no es una botella que hay que llenar, sino un fuego que es preciso encender."

Montaigne, citado por Savater (1997).

El escenario mundial en los últimos diez años, primordialmente desde finales del siglo XX hasta inicios del siglo XXI, ha mostrado cambios significativos reflejados en todos los órdenes de la actividad humana, por ejemplo, la globalización económica, el desarrollo científico tecnológico acelerado, la comunicación tecnológica de vanguardia posmoderna, movimientos migratorios, conciencia global para la protección ambiental y la agudeza de patologías sociales como drogadicción, delincuencia y desintegración familiar, entre otros.

En este sentido, las demandas necesarias de cambio planificado para producir la diferencia deseada exigen a la institución educativa armonizar las exigencias del entorno comunal, regional, nacional y mundial mediante la oferta educativa; esta constituye uno de los principales dominios de decisión y desarrollo en las sociedades evolucionadas, implicando por supuesto, una reflexión en la orientación curricular establecida y la "deseada" como un medio para mejora e innovador.

Por ello, este artículo, resultado de una investigación bibliográfica, propone 


\begin{abstract}
This article proposes an approach to education from the perspective of the theory of multiple and emotional intelligences. Intelligence is understood as the capacity to solve problems or create valuable products for a certain community or culture. Therefore, success or failure at school could be predicted based on the child's capacity to solve problems and adapt to different social or natural sceneries. From this perspective, education is seen as a social structure that benefits the accommodation mechanisms which explain, predict, interpret, clarify and transmit culture and social practices. These mechanisms give shape to the classroom-a space for teacher-student interactions. As a result, the symbolic focus of education offers an opportunity for conducting research which values cultural diversity, the student's learning context, the stimulus given to individual intelligences and the changing human interactions. In this way, a more altruistic solution to problems is provided.
\end{abstract}

Key words: Curriculum, intelligence, symbolic focus, diversity, multiple intelligences, emotional intelligences, classroom, context, education. inicialmente el enfoque de los sistemas simbólicos, basado en las teorías de las inteligencias múltiples y emocionales, vinculadas con las concepciones curriculares en el sistema educativo costarricense, ofreciendo al lector un contexto oportuno y objetivo de la relación existente entre ellas.

Compartir estos conceptos es esencial para la comunicación de ideas, puntos de vista, problemas y soluciones dentro de cualquier temática, en este caso, mediante una discusión reflexiva de los postulados teóricos y su relación con el contexto de la educación costarricense fundamentando un marco referencial para una valoración coherente.

Es decir, necesariamente se debe tener un punto de partida de la concepción de inteligencia, así como de los principios que citan su progenitores en el desarrollo de sus propuestas teóricas que se expondrán a lo largo del artículo.

El documento diluye las inteligencias múltiples con referencia al currículo y los fines como objetivos de la educación costarricense debido a que la adquisición del conocimiento es fundamental en el sistema educativo formal de nuestro país.

Por lo cual, uno de los elementos que facilita y promueve el cambio, el desarrollo educativo y profesional es el currículo escolar; rescatar la importancia del currículo para mejorar la calidad de la enseñanza, debe llevarnos a descubrir el sentido de la cultura en las escuelas y escrutar las formas de su realización práctica.

\section{Currículo en el enfoque de los sistemas simbólicos de las inteligencias múltiples}

Currículo proviene del latín currere haciendo referencia al concepto de carrera, al recorrido que debe ser realizado, en este caso, por la institución educativa (Sequeira, 2000).

Grundy (1991) indica que “(...) el currículo, no es un concepto, sino una 
construcción cultural. No se trata de un concepto abstracto que tenga algún tipo de existencia fuera y previamente a la experiencia humana. Es un cambio, una forma de organizar un conjunto de prácticas educativas (...)" (P. 20-21).

En este sentido, la reproducción cultural no puede estar fuera de la experiencia humana, Freire (citado por Sequeira, 2000) argumenta que "cultura" es el aporte del ser humano al mundo como un resultado de su trabajo, de su esfuerzo creador y recreador, al no dejar de lado la búsqueda de alternativas ante diversas situaciones que originan en su instinto creador su respuesta o previas soluciones, ejemplo de ello es cuando una persona no encuentra respuesta ante una enfermedad en la medicina convencional recurre en última instancia a la medicina alternativa (medicina natural u homeópata).

El currículo no es abordable desde una sola perspectiva, debido a que forma parte de la interpretación de las diversas realidades y la construcción del conocimiento, adquirido como “(...) una interacción dialéctica teórica y práctica como una adquisición de una reconstrucción cíclica e histórica de cada individuo" (García, Álvarez, Guerrero, Desantes, Fernández, Losado, García, Marín, Martínez, Pérez, y Soler, 1996, p. 238).

Gimeno (1996) opina que esas "perspectivas" no pueden ser indiferentes a las complejidades de la práctica pedagógica, o sea, el docente promueve en el estudiante un aprendizaje significativo, donde el papel que desempeña en los procesos de enseñanza y aprendizaje concretan el currículo.

Desde este punto de vista, un currículo antes de ser objeto de teorización se debe construir en torno a los problemas cotidianos que se dan en el ámbito escolar y que afectan a las personas involucradas en un contexto educativo particular.

Torres (1996) plantea una dimensión del currículo más de cara al trabajo práctico y reflexivo, facilitando la elaboración de un currículo integrado, como el propuesto mediante el enfoque de los sistemas simbólicos de las múltiples inteligencias, que favorece las condiciones de motivación por el aprendizaje, la comprensión de la realidad y, sobre todo, la "significatividad" de la experiencia aprendida.

Argumenta el autor, que en el sistema escolar visiblemente existe ausencia de una verdadera reflexión sobre la situación y los orígenes que enfrentan los grupos sociales minoritarios y marginales, silenciados en los acontecimientos históricos, socioeconómicos y culturales que marcan a los diferentes grupos, como el caso de los emigrantes nicaragüenses en nuestro país, los aborígenes de la zona atlántica, que no son contemplados en el currículo oficial.

La educación es ante todo, una acción política y ética, por lo cual la institución escolar cumple una función de contribuir a la reconstrucción de la cultura, cuyo propósito es la internalización de sus elementos y rasgos fundamentales de la cultura, como parte de una educación inclusiva que procura integrar a todos; es una forma de asegurar a ricos y pobres el acceso a la promoción humana. Incluir implica una visión totalizadora y reflexiva del currículo, pensado dentro de un contexto social, que evidentemente se relaciona con ampliar la visión de la inteligencia lingüística y lógica matemática a múltiples inteligencias.

Castillo (2003) explica la concepción de currículo como una experiencia integrada y de realización personal, cuya función fundamental es la organización de experiencias personales integradoras y altamente de satisfacción para los aprendizajes individuales de cada sujeto, situando la atención en la persona y en la construcción de un proyecto personal de vida libre, responsable y auténtica.

Es decir, el papel del educador consiste en estar al servicio del grupo de alumnos y dejar en manos de los aprendices (estudiantado) muchas de las decisiones, actividades y funciones que se han delegado al desempeño profesional. 
Antúnez (1995) señala que el aprendizaje del estudiantado debe ser significativo, siendo este lógico del contenido y organizado coherentemente, al establecer una conexión entre el contenido a trabajar y la estructura mental del aprendiz, o sea, sus conocimientos previos, su nivel de desarrollo y su estilo estratégico de aprender.

Por ello, el nivel de desarrollo del alumno puede llegar a ser el que determine cuáles conocimientos son asimilados mediante el tipo de inteligencia que posee el sujeto o el establecimiento de relaciones significativas de lo conocido (asimilado y acomodado) con lo nuevo por conocer (reestructuración del pensamiento).

En este sentido, un currículo personalizado e integral contempla el quién, qué y cómo enseñar, vinculando las inteligencias múltiples como adquisición sociobiológica en cada ser humano que predispone a los sujetos a crear y recrear su medio.

En general, se concibe el currículo como el medio, mediante el cual los estudiantes aprenden a enfrentar los asuntos y las metas sociales, buscando capacitar al ser humano para mantenerse y funcionar con eficiencia en un mundo de rápido cambio, que demanda personas críticas, reflexivas y multifacéticas con altas exigencias de una inteligencia intrapersonal e interpersonal, para producir una diferencia del "ser al debería ser".

\section{La teoría de las inteligencias múltiples: concepto y fundamentos}

La teoría de las inteligencias múltiples se organiza desde una perspectiva biológica, expone que la inteligencia es la capacidad para resolver problemas o conflictos.

Sin embargo, la tendencia biológica a participar de forma concreta en la resolución de problemas tiene que asociarse también al entorno cultural. Por ejemplo, la teoría de las múltiples inteligencias es susceptible al contexto, como un sistema computacional basado en las neuronas, donde cada inteligencia se activa o se "dispara" a partir de ciertos tipos de información, presentada de forma interna o externa.

Es importante que se comprenda "sistema simbólico" como un sistema de significado, producto de la cultura, que captura y transmite formas importantes de información.

No obstante, las inteligencias, involucran asimilar y acomodar en el individuo la relación de sus facultades o competencias físicas y mentales otorgadas por su entorno sociocultural y la predisposición biológica como especie, implicando la mezcla de varias de ellas para disponer al sujeto para aprender un conocimiento general o específico sobre su medio.

El autor, agrupó la variedad de habilidades humanas en siete categorías: inteligencia lingüística, lógico matemática, espacial, corporal-cinética, musical, personal (interpersonal, intrapersonal) y naturalista, mediante el reconocimiento de las mismas en fases.

\section{Fases de la inteligencia múltiple}

Gardner (1994) menciona siete fases o etapas que han caracterizado el estudio de la inteligencia. En cada una considera tanto las opiniones de la comunidad científica como las implicaciones dentro del mundo de la práctica educativa.

1. Concepciones profanas de la inteligencia: a través del tiempo, la palabra inteligencia no se ha usado de forma precisa, pues se utilizó con el fin de describir poderes mentales; las personas pertenecientes a la cultura occidental se consideraban "inteligentes" si eran ingeniosas, científicamente astutas o sabias.

2. El giro científico: al escuchar sobre el primer test de inteligencia y del 
concepto de cociente intelectual, muchos psicólogos y educadores se percatan del enorme potencial de esta invención, creando instrumentos más perfeccionados. El trabajo en el ámbito del desarrollo de los tests de inteligencia continúa hasta nuestros días.

3. La pluralización de la inteligencia: muchos estudiosos decidieron creer que la inteligencia era un "constructo" unitario, con el tiempo se pudo determinar que de la misma manera en que las personas difieren en peso y altura, en integridad, en honradez y en otros aspectos; también difieren en lo listas que son y en las diferentes inteligencias que poseen $o$ sus manifestaciones, según las demandas de habilidades y destrezas que las circunstancias del medio les exijan.

\section{La contextualización de la inte-} ligencia: la inteligencia o las inteligencias son siempre una interacción entre las tendencias biológicas y las oportunidades de aprendizaje que existen en una cultura.

\section{La distribución de la inteligen-} cia: reconoce que casi nunca las personas productivas trabajan solas simplemente usando su cabeza; lo normal es que los individuos trabajen con todo tipo de objetos humanos e inanimados o protésicos (artificial), llegando a formar parte integral de sus actividades, que parece lógico considerarlas un armamento intelectual de cada individuo.

\section{La individualización del con-} cepto de inteligencia: la mente de cada individuo es diferente, cada una tiene su contexto sociocultural peculiar y cada mente participa de varias extensiones humanas y no humanas.

\section{La educación de las inteligen-} cias: se refiere a una educación significativa donde el alumno usa los conceptos y la habilidades adquiridas en la escuela para resolver problemas, sean nuevos o desconocidos e incluso ejecutar nuevos proyectos, manifestando en este proceso lo comprendido y no solamente imitando los aprendizajes de un conocimiento general o particular que se ha suministrado.

Un aspecto que debe de quedar claro antes de iniciar con los fundamentos teóricos de las inteligencias múltiples, es la afirmación del autor al describir las inteligencias individuales e incluso las primeras etapas del desarrollo simbólico que constituye una función principalmente útil para propósitos científicos; ni las diversas inteligencias, ni las variadas corrientes existen en aislamiento prístino (antiguo). Es decir, más bien, estos "sistemas ideales" siempre se encuentran en un ambiente cultural, que llega a ejercer control decisivo de su curso (Gardner, 1993, p. 366).

Por ejemplo, el papel social que puede cumplir una persona con una predominante inteligencia espacial, puede variar desde ingeniero, arquitecto, artista, escultor, diseñador... En tanto que una persona que ejerza una profesión como la abogacía puede comprender una combinación de inteligencias lingüística, lógica e interpersonal.

Lo mismo sucede con el concepto de "número de inteligencias" debido a que

(...) no existe, y jamás puede existir una sola lista irrefutable y aceptada en forma universal de inteligencias que pueden avalar todos los investigadores. Podemos aproximarnos más a esta meta si nos atenemos sólo a un nivel de análisis o meta (...); pero si estamos buscando una teoría decisiva, del rango de la inteligencia humana, podemos espera que jamás completaremos nuestra investigación (Gardner, 1993, p. 95).

La inteligencia se comprende como “(...) la capacidad de resolver problemas, o de crear productos valiosos en uno o más ambientes culturales" (Gardner, 1995, p. 10) considerando algunos prerrequisitos de una inteligencia, como asegurar que una inteligencia humana es genuinamente útil e importante, al menos en determinados ambientes culturales y abarca una razonable gama completa de clases de habilidades que valoran las culturas humanas. 
Con el fin de presentar un fundamento teórico sólido a las afirmaciones de Gardner, Chaverrí y Navarro (2002) citan unas ciertas "pruebas" que cada inteligencia debería cumplir para poder ser visualizada en todo el sentido de la palabra y no considerarse solamente como un talento, una habilidad o aptitud.

De esta manera, algunos criterios de una inteligencia pueden ser según el autor

\section{Aislamiento por daño cere-} bral: se estudian las funciones aisladas del cerebro a través de casos de lesión del cerebro y de la enfermedad degenerativa, se pueden identificar las localizaciones neurofisiológicas reales para las funciones específicas del cerebro. En este sentido, una inteligencia tendrá su función identificada en una localización específica en el cerebro humano.

2. La existencia de "idiots savants" (idiotas sabios), personas prodigiosas y otros individuos excepcionales: ellos muestran un perfil muy disparejo de habilidades y deficiencias. Pueden ser prodigios al estilo de Mozart que puede tocar el piano a la edad de cuatro años o el criterio de niños que presentan un desempeño de una habilidad particular contra un desempeño humano deficientes, por problemas de discapacidades, aquí aplican casos de autistas al estilo de "Rainman" de Dustin Hoffman, que realizó cálculos matemáticos impresionantes, indicando exactamente que las capacidades humanas específicas pueden demostrarse en altos grados en casos únicos.

\section{Operación medular o conjunto} de operaciones identificables: conjunto identificable de los procedimientos y las prácticas únicos a cada inteligencia; existen una o más operaciones y mecanismos básicos de procesamiento de la información identificables y separados.
4. Historia distintiva de desarrollo, junto con un conjunto definible de desempeños expertos de "estado final": contempla un modelo claro de historia de desarrollo, documentada en la cognición humana, una inteligencia tiene un conjunto identificable de etapas del crecimiento con un nivel que va desde el novicio, por el que atraviesa la mayoría hasta llegar al nivel de un talento que existe como estado del extremo en el desarrollo humano. Es importante, citar que las inteligencias no son aisladas y todas tienen un nivel inicial que luego se van descomponiendo en etapas de crecimiento desiguales.

\section{Historia evolucionista y la evo-} lución verosímil: a medida que los antropólogos culturales continúan estudiando la historia de la evolución humana, se evidencia que en nuestra especie se ha desarrollado la inteligencia en un cierto plazo, según la adquisición en la experiencia humana.

6. Apoyo de tareas psicológicas experimentales: identifican conjuntos de las habilidades modulares o específicas para diversos dominios del comportamiento humano, pueden interactuar en la ejecución de tareas complejas, o sea, una inteligencia se puede identificar por las tareas específicas que pueden ser realizadas, observadas y medidas.

\section{Apoyo de hallazgos psicométri-} cos: el uso de instrumentos psicométricos para medir la inteligencia como "Coeficiente Intelectual" (C.I.) se ha utilizado tradicionalmente para medir solamente tipos específicos de capacidad; pueden proporcionar una pista para una inteligencia, específicamente en el aspecto de lingüística y lógico matemático. En la medida que las tareas supuestamente evalúan una inteligencia, se correlacionan o no entre sí, puede ser indicador de apoyo a una inteligencia.

8. Susceptibilidad a la codificación en un sistema simbólico: los seres 
humanos han desarrollado muchas clases de sistemas simbólicos para representar y comunicar el conocimiento humano. Una inteligencia tiene su propio conjunto de las imágenes que utiliza, únicas e importantes para ejecutar un conjunto identificado de tareas en un contexto específico.

Cabe señalar que el autor efectúa dos aclaraciones importantes a la hora de definir una inteligencia: no son equivalentes a sistemas sensoriales, ya que una inteligencia no depende de un sistema sensorial exclusivo, sino que actúan con varios de ellos (aunque no necesariamente con todos) $y$, las inteligencias operan con sus propios procedimientos y bases biológicas. De esta manera, es un error tratar de hacer comparaciones entre ellas, ya que cada una tiene sus propios sistemas y reglas.

Las inteligencias son una muestra representativa de las potencialidades del ser humano, las cuales en el contexto de la alta tecnología e investigación no pueden concebirse como un producto acabado. Por lo cual las siguientes propuestas de las inteligencias estudiadas por Gardner (1993), son posiblemente las más empleadas en los centros educativos y otras, por el contrario, son relegadas o vistas como algo sin importancia en la formación del educando.

En este sentido, comprender que no se pretende clasificar a los niños y a las niñas de una institución en una inteligencia determinada, más bien se busca que el docente impulse estas potencialidades para el adecuado desarrollo del infante de una manera integral.

Prieto (citado por Moreno, 2000; Enero-Marzo) afirma que todo acto educativo es un acto comunicativo, con base en una institución educativa modeladora de la comunicación clásica. Sin embargo, es una simple visión cerrada y obtusa de una escuela que tiene el conocimiento y lo transmite a los educandos; es una posición descontextualizada y evidentemente relega la interacción de los cambios continuos y latentes de la sociedad.
Consecuentemente, el sistema educativo costarricense da prioridad a la inteligencia lingüística y lógico matemática, abandonando la constante y creciente demanda social en el mercado de individuos multifacéticos, que sean capaces de poder ejercer diversas actividades que requieren de otras habilidades y capacidades relegadas por nuestro sistema como son la inteligencia corporal-cinética, la musical y la espacial.

\section{El enfoque de los sistemas simbólicos de las inteligencias múltiples y la inteligencia emocional en el contexto costarricense}

Iniciar la discusión sobre la teoría de las inteligencias múltiples implica ofrecer un pequeño espacio de familiaridad con sus principios básicos; por ejemplo, esta teoría tiene su desarrollo aproximado en los años cincuenta del siglo pasado con Gulford (citado por Klingler y Vadillo, 1999), quien creó un modelo multifactorial de 120 factores, que abrió la puerta a la concepción de las inteligencias múltiples en Norteamérica.

Cabe citar que existe una variedad de teorías y concepciones sobre las inteligencias; las más populares en la actualidad han sido los postulados de Sternberg, quien a finales de los ochenta realizó un simposio sobre la inteligencia y la evaluación asignando importancia a las funciones ejecutivas, así como al modelo de Gardner y Goleman, con una variedad de inteligencias, entre ellas algunas no tradicionales como la musical y las inteligencias personales (intrapersonal e interpersonal).

De acuerdo con las investigaciones, el psicólogo Gardner (1995) definió inteligencia como la capacidad para resolver una situación conflictiva o elaborar productos valiosos en un determinado contexto comunitario o cultural. Por lo tanto, el éxito o fracaso escolar se podrán predecir dependiendo 
de la capacidad del niño para resolver diferentes situaciones.

En concordancia con el autor, se considera no válida la determinación de la inteligencia de una persona fuera de su propio ambiente, a la que se solicita que haga algunas tareas nunca efectuadas por ella e incluso, que no querría repetir.

La inteligencia como un potencial biopsicológico, en otras palabras, señala que todos los miembros de la especie poseen el potencial para ejercer un conjunto de facultades intelectuales de las que la especie es capaz.

El enfoque de los sistemas simbólicos fundamenta la comprensión de la teoría de las inteligencias múltiples, al brindar especial significado a los sistemas simbólicos de comunicación reflejados en distintas formas como el arte, el lenguaje y la ciencia entre otros en el contexto cultural, donde el sujeto se desenvuelve.

Gardner (1995, p. 350) trabajó con la definición de su mentor Goodman y de otros, y señala la siguiente definición de símbolo:

(...) toda entidad (material o abstracta) que pueda denotar o referirse a otra entidad. Según esta definición, fácilmente se consideran como símbolos las palabras, cuadros, diagramas, números y una serie de otras entidades (...) es cualquier elemento (...) en tanto que se emplee para representar cierta clase de información (y así se interprete).

Chaverrí y Navarro (2002) afirman que la actividad humana es un proceso sociogenético, como lo expuso Vygostky, resultado del aprendizaje social, de la interacción de signos sociales, de la cultura y de las relaciones mismas.

En este sentido, la cultura regula y organiza el pensamiento, englobando los conceptos que son parte de la experiencia y del conocimiento del ser humano.

Por tanto, la posición epistemológica de "educación", citada por Grundy (1994) como Ciencias de la Educación, es oportuno mencionarla en relación con comentado anteriormente
“(...) explica, predice, interpreta y transmite la cultura y la práctica social como un fenómeno interaccional transformador" (p. 143).

Es decir, educar implica transformar la sociedad, de allí que sea un proceso complejo, dinámico y comprometido. Complejo debido a que su insumo son los seres humanos. Dinámico y comprometido porque como hecho sociohistórico se ubica en circunstancias y contextos sociales particulares a los que pretende y debe dar respuestas (Carr y Kemmis, 1988).

De alguna manera, la relación entre cultura y educación comprende el marco de un contexto, García, et al. (1996, p. 226) como un “(...) conjunto de elementos físicos, espaciales, temporales, sociales y culturales" que configuran los escenarios en que tienen lugar los procesos de enseñanza-aprendizaje. Igualmente sostiene Frederick Erickson y Jeffrey Shultz (1981) quienes apuntan que contexto es "(...) lo que la gente hace, dónde, cuando y cómo lo hacen."

Resalta también la participación de algunos investigadores como Feldman, Olson, Salomón que intentan darle una nueva visión a los fundamentos de Piaget, incluyendo una nueva lectura de los sistemas simbólicos lingüísticos, lógico y numérico y la consideración de sistemas simbólicos como el musical, corporal, espacial y los personales. En este caso, “(...) el reto consiste en componer un retrato del desarrollo de cada una de estas formas de competencia simbólica y determinar empíricamente qué conexiones o distinciones pudieran obtenerse con ellas" (Gardner, 1993, p. 58).

Feldman (citado por Gardner, 1993), establece que este enfoque pluralista (distinto del piagetiano unilineal) plantea el desarrollo de la cognición con base en una serie de dominios, entre ellos el universal (todos los individuos del mundo tienen conocimiento, sea por pertenecer a la especie o por necesidad de enfrentar el ambiente físico y social que aprenden a confrontar 
y dominar, como el sistema simbólico lógico-matemático); el cultural (dominio determinante en una cultura y en otra; tiene un valor mínimo o irrelevante, por ejemplo, la lectura o conocimientos astronómicos para navegar de noche, dominios que son propios de un grupo dentro de la misma cultura como jugar ajedrez o el juego japonés "go", conocimiento de las raíces generacionales por los israelitas y musulmanes de gran importancia en el contexto religioso) y los singulares, que explican como una persona o un grupo pequeño de individuos logra tener la habilidad o habilidades necesarias para una actividad determinada, como aquellas personas que en un momento tienen un conocimiento "único", como es el caso de Dalai Lama y su representación en el budismo.

Lo interesante es que estos dominios plantean un progreso de lo singular a lo general, es decir, lo que un individuo sabe en un determinado momento es posible que lo conozca y domine un grupo o una cultura en el futuro, como la trayectoria histórica ejemplificada en el caso de Francia, cuna de la educación mundial: Napoleón y Juana de Arco.

El dominio es un progreso postulado por etapas que va desde el nivel inicial o de novicio, pasando por "aprendiz" a la etapa de "experto", siendo el primero un sujeto que adquiere habilidades y destrezas según el contexto en que se desenvuelve, sin perder de vista sus capacidades innatas, como lo plantea el constructivismo biológico y social y, de esta manera, ser un "experto" un individuo con la facilidad de poder desenvolverse dentro de las exigencias sociales en que se ve inmerso, por ejemplo, Feldman (citado por Gardner, 1995) ha estudiado a los niños prodigio, quienes se encuentran en un dominio particular.

En el Proyecto Cero de Harvard se utiliza una perspectiva desarrollista $\mathrm{y}$ neuropsicológica para entender la estructura y organización del funcionamiento simbólico humano; Gardner y sus colegas apuntan que "(...) la meta ha sido conocer las "clases naturales" de sistemas simbólicos: las familias de sistemas simbólicos que se mantienen unidas (o que se descomponen) y las formas en que pudieran representarse en el sistema nervioso humano" (Gardner, 1995, p. 61).

La definición y delineamientos de sistemas simbólicos se basan en: a) las consideraciones lógicas de los estudiosos de sistemas simbólicos específicos; b) las históricas o culturales para designar dominios o sistemas simbólicos particulares para fines educacionales o de comunicación en una cultura; las empíricas de probadores de inteligencia, por medio de correlaciones estadísticas y, d) las neuropsicológicas contemplando cuales capacidades simbólicas fallan juntas en una lesión cerebral suponiendo la correspondencia a la misma clase natural.

La teoría se visualiza de una forma interdisciplinaria, o sea, el fundamento de cada inteligencia se realiza desde estudios neuropsicológicos y antropológicos; aunque efectivamente existe una inclinación del autor por lo biológico.

Sin embargo, el planteamiento del enfoque de sistemas simbólicos implica una correlación entre ambas disciplinas, dando una respuesta a un continuo entre la constitución y los procesos del sistema nervioso, presente en una experiencia determinada para un organismo y, este puede comprender y tratar con entidades simbólicas como palabras, oraciones, números...; hasta llegar a los niveles de competencia humanos de cada cultura.

Los símbolos pueden denotar o representar e incluso transmitir estados de ánimo, sentimientos o tonos tal y como la comunidad pertinente escoja interpretarlos, como es señalado en la inteligencia emocional de Goleman (1995).

Además, es indispensable aclarar que los símbolos solamente interpretan entidades significativas, pero estas a su vez se convierten en elementos de un sistema mucho más elaborado, ejemplo, los patrones de movimiento y gestos son símbolos para 
un sistema simbólico llamado "danza", los acordes y notas musicales son símbolos de un sistema llamado "sinfonía".

De esta manera, los símbolos adquieren su máxima utilidad cuando entran en el diseño de estos productos simbólicos completos para transmitir un conjunto de significados que otros miembros de la cultura pueden comprender, interpretar, apreciar, criticar y transformar.

Las personas tratan de dominar diversos sistemas simbólicos para el desarrollo dentro de su propia cultura y estos a su vez evolucionan o no con las transformaciones que los individuos ejecutan.

La aparición de la competencia simbólica, según el gestor de la teoría de las inteligencias, se da en etapas de desarrollo, corrientes de simbolización, ondas de simbolización y canales de simbolización (estas tres últimas se desarrollan en la niñez temprana o edad preescolar).

Chaverrí y Navarro (2002) resumen las etapas de desarrollo planteadas en la teoría de las inteligencias múltiples

1. Infancia: se adquieren determinados entendimientos básicos, que se utilizarán en el uso posterior de símbolos.

El recién nacido por ejemplo, tiene una serie de mecanismos para el reconocimiento de actividades placenteras y no placenteras: como mamar y mirar; en un inicio estos mecanismos $\mathrm{u}$ habilidades inciden sobre todos los objetos, pero luego se inicia un proceso de discriminación de ciertas actividades más productivas que otras.

En esta parte el "significado" va unido a la conducta; por supuesto, esto va influenciado por la interpretación que los adultos hacen de su conducta. Ya para el primer año, llega a reconocer que los individuos pueden cumplir determinados papeles con la conducta asociada (dar de comer); por ejemplo que los acontecimientos tienen consecuencias (si tira el chupón, caerá al piso), que existen categorías de objetos (muñecas son distintas a las flores) y también puede, como veremos más adelante, existir un reconocimiento de tonos, apreciar el espacio a su alrededor, utilizar su cuerpo para realizar distintas acciones, reconocimiento visual, auditivo y oral de objetos (mamí, leche, pan) y, además, la combinación de estas para realizar otras actividades.

2. Niñez temprana: (dos a los cinco años): se desarrolla la simbolización básica, es decir, cuando el infante puede apreciar y crear instancias de lenguaje (oraciones e historias), la simbolización bidimensional (cuadros), simbolización tridimensional (barro y bloques), simbolización de gestos (danza), música (cantos), drama (juego de simulación) y determinadas clases de entendimiento matemático y lógico, incluyendo la apreciación de las operaciones numéricas básicas y explicaciones causales sencillas.

3. Edad escolar: habiendo logrado cierta competencia básica en la simbolización, el niño y la niña procede a adquirir niveles más altos de simbolización, apreciados culturalmente. También en este tiempo, domina diversos sistemas simbólicos notacionales o de "segundo orden", que le serán útiles para realizar distintas tareas culturales.

4. Adolescencia y adultez: el individuo puede utilizar competencias simbólicas y transmitir el conocimiento simbólico adquirido.

Algunas corrientes de simbolización son la progresión singular en cada uno de los sistemas simbólicos específicos; continuando con el ejemplo de la música, implica resolver las relaciones básicas con el tono que se obtiene dentro de una escala. Este desarrollo es la articulación de una inteligencia particular; luego que se ha vuelto susceptible a involucrarse en los sistemas simbólicos de la cultura: aspectos medulares de la inteligencia musical (tono y ritmo), son ordenados por aspectos simbólicos de la música como la expresión (es 
una pieza alegre) y la referencia (es una selección anterior de la canción) (Chaverrí y Navarro, 2002).

Es decir, las ondas de simbolización se refieren a los procesos que inician dentro del terreno simbólico particular, pero por su propia naturaleza, se extienden con rapidez y a veces en forma inapropiada a otros dominios simbólicos como la estructuración del papel o sucesos (habilidad del niño de 2 años para indicar que se ha realizado una acción o que un agente ha desempeñado un papel). Por ejemplo, el lenguaje y el juego de "simular" son "lugares propios" del conocimiento estructurado de sucesos, ("mami dormida": acuesta la muñeca).

No obstante, sin importar en que dominio simbólico esté una tarea o actividad dada, es probable que el niño de esta edad traslade dos estructuras de conocimiento ("pinta un carro": el niño toma el lápiz y lo desliza diciendo "ruumm"), de esta forma el símbolo cobra sentido en el papel o suceso.

La transformación analógica o topológica, es cerca de los 3 años y consiste en el empleo que da el niño al símbolo, capta dentro del propio vehículo simbólico algunas relaciones observadas originalmente en el campo de referencia que está simbolizando. Una retorcida línea en el papel, realizada por el niño, puede convertirse en una persona, por ejemplo. Esta onda se inclina por formas, tamaños o valencias relativas (González, 2004).

En esta transformación digital o cuantitativa cercana a los 4 años, se procura que el número de elementos en una producción sea el correcto, pero al estar tan concentrado que estos elementos sean correctos, a veces pierde aspectos esenciales de la producción (ejemplo: canta una y otra vez una canción desde el inicio hasta que tenga los elementos adecuados).

Cabe mencionar, cómo argumentan Hernández y Redondo (2002) que los canales de simbolización principian con la notación al inicio de la edad escolar (5 a los 7 años) y, es la capacidad para inventar diversos sistemas de notación que, en forma de "segundo orden", se refieren a los sistemas simbólicos básicos, como las letras por el lenguaje hablado, números, mapas, diagramas, claves, sistemas notacionales musicales o de danza que fueron diseñados para captar los puntos destacados de un despliegue simbólico.

Curiosamente, la teoría de las inteligencias múltiples se describe de la manera más exacta como "una filosofía de la educación", es decir, una actitud hacia el aprendizaje, una meta-modelo educacional $\mathrm{y}$, al mismo tiempo, no es un programa de técnicas y estrategias fijas.

De este modo, ofrece a los educadores una oportunidad muy amplia para adaptar de manera creativa sus principios fundamentales a cualquier cantidad de contextos educacionales. Stainback y Stainback (1999) opinaron

Los niños difieren en muchas dimensiones y la identidad de cada uno está configurada por su pertenencia a muchas grupos (...). La creciente diversidad racial y étnica de nuestra sociedad y nuestras escuelas obliga a que los programas y currículos escolares tengan en cuenta las diferencias entre los niños. (P. 38).

En este sentido, la socialización no está dada únicamente bajo el soporte de dos únicos sistemas simbólicos (la lógica matemática y verbal), que vienen a interactuar en la construcción del conocimiento, a pesar de que la escuela no los ha reconocido, como los sistemas notacionales, entendidos como procesos de representación, cuya unidad básica es el símbolo, que corresponden a datos de entrada y operacionalidad como representaciones mentales que se reflejan, por ejemplo, en el movimiento, la música y la imagen (Chaverrí y Navarro, 2002).

Encontrando referencias, que citan los objetivos de la educación resaltando su finalidad al contribuir en la formación de la personalidad en un medio que favorezca su desarrollo físico, intelectual y moral; así como desarrollar el pensamiento y preparar para la vida, una guía en la adquisición de una cultura general permite en 
este contexto justificar la necesidad de lo que trato de exponer, al sustentar los conceptos de inteligencia múltiple, refiriéndome a lo que su progenitor, el psicólogo Gardner (1993) explica como comprender la realidad humana, al deber intentar visualizarla como un hecho de distintos dominios, y cómo es necesario utilizar múltiples modos de conocer, crear productos y solucionar problemas para comprender el mundo en que vivimos.

Actualmente en nuestro país el Centro Nacional de Didáctica (CENADI) y la Fundación Omar Dengo desarrollan un proyecto fundamentado en la "Teoría de la Comprensión" que parte del empleo de los principios de estas teorías norte- americanas, la utilización de implementos tecnológicos en el desarrollo de destrezas y habilidades cognitivas como es la informática y el uso de mapas conceptuales en primaria (Centro de Innovación Educativa y Fundación Omar Dengo, 2003).

Igualmente en el Instituto Educativo Moderno (IEM), Camacho (2003) construyó, mediante su experiencia como educadora en ejercicio en primaria, una malla para la planificación de los contenidos y objetivos de las asignaturas, distribuido en columnas como muestra a continuación el cuadro, para acercarse a las postulaciones de las múltiples inteligencias y ejecutarlos en un centro educativo privado.

Cuadro 1

Malla de planificación de los aprendizajes en primaria a través de los enfoques del sistema simbólico de las múltiples inteligencias

\begin{tabular}{lllllll}
\hline Objetivo por área & Kinestésica & Matemática & Musical & Lingüística & Espacial & Evaluación \\
\hline Español: & Dramatizar & Ordenar las & Leer y & Leer en & Ilustra las & Mediante \\
Enriquecer la & oraciones & oraciones y & cantar las & forma & oraciones y & la lectura \\
lectura con el & creadas por & el vocabulario. & oraciones en & individual & el vocabulario & oral, la \\
reconocimiento & el alumno. & & diferentes & y grupal, & creado por & observación \\
de sílabas & & & velocidades. & oraciones & el alumnado. & y una hoja \\
nuevas. & & & y trozos. & & de cotejo.
\end{tabular}

Construir oraciones.

La educadora presenta al mismo tiempo una propuesta en la evaluación, mediante una serie de criterios por valorar en cada una de las inteligencias que se ejemplifica y detalla en el siguiente esquema.

Cuadro 2

Esquema para valorar a los aprendices en los contenidos de Primer Grado

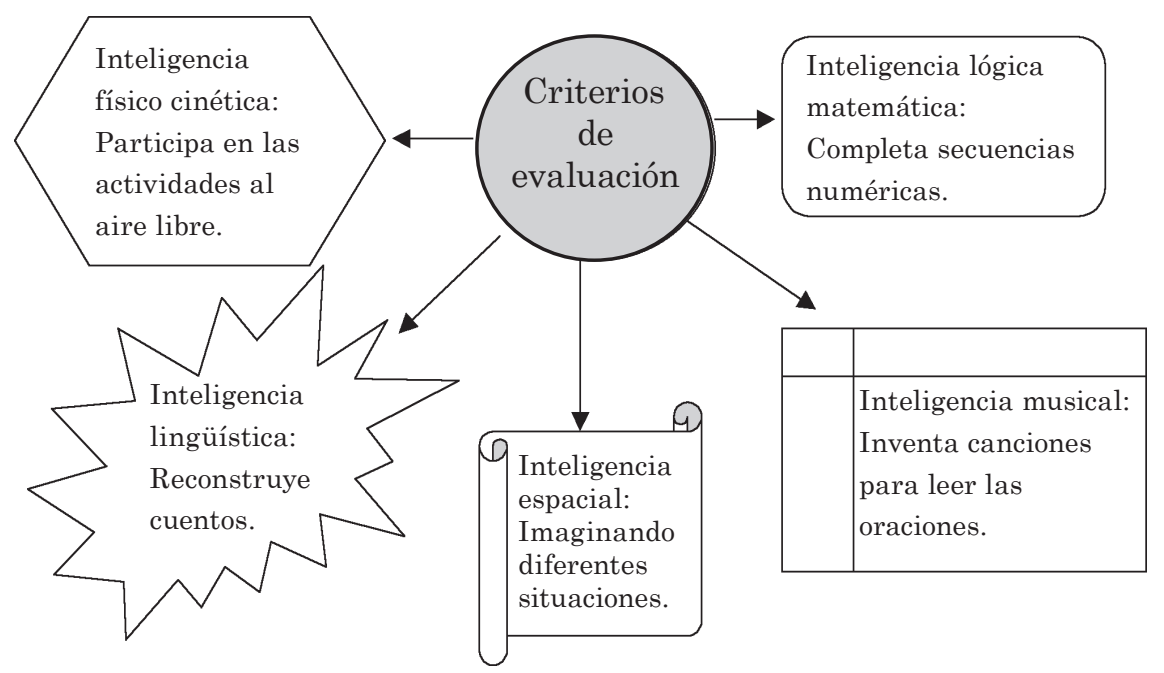


Los fines y objetivos de la educación costarricense en el enfoque de los sistemas simbólicos de las inteligencias múltiples y la inteligencia emocional

Nuestra raíz cultural esta clarificada en los fines de la educación; de acuerdo con Kingler (1999) es necesario comprender cómo la cultura se transmite y se crea a través de la educación; es decir, se entiende mediante el lenguaje que es representado por conocimientos, habilidades, experiencias y una memoria histórica y acumulativa en una sociedad, siendo uno de los principios fundamentales dentro de la "psicología cognitiva”.

En Costa Rica, según la Ley Fundamental de Educación determinada en setiembre de 1957, se refleja la ideología democrática costarricense, que muestra una aspiración de justicia hacia la sociedad, intentando sostener un equilibrio o conciliación entre los intereses del individuo y los de la colectividad.

Los humanos como especie, han desarrollado complejas culturas, formas de organización social, debido a su naturaleza y capacidad de comprender y comunicarse simbólicamente, visualizado en valores, normas y estructuras sociales. Dentro de estos "ecosistemas", comprendidos como estructuras sociales, se cita primeramente la familia como la organización social que busca el ajuste del ser humano en el medio ambiente, es la unidad o "casa", que desempeña las funciones socio culturales que para Kingler (1999) se ejemplifican en la reproducción, protección, apoyo, alimentación, socialización y transmisión de la cultura.

A pesar de la necesidad de que la Asamblea Legislativa apruebe una nueva ley acorde con la situación actual del país y los cambios transformadores de nuestro medio cultural, que aporte un sentido de modernización cualitativa al sistema educativo, no se pueden invalidar los fines de la educación por su época de origen, que más bien proyectan el espíritu democrático de nuestra raíz social y un mapeo de nuestros valores citados en la Ley en el Código de Educación en 1969 en las páginas 446 y 447 (citado por Dengo, 1998).

En este sentido, los fines de la educación costarricense se destacan por fomentar: a) la formación de ciudadanos amantes de su Patria, conscientes de sus deberes, de sus derechos y de sus libertades fundamentales, con profundo sentido de la responsabilidad y de respeto a la dignidad humana; b) la contribución al desenvolvimiento pleno de la personalidad humana; c) la formación de ciudadanos para una democracia en que se concilien los intereses del individuo con los de la comunidad; d) estimular el desarrollo de la solidaridad y de la comprensión humana y, e) conservar y ampliar la herencia cultural, impartiendo conocimientos sobre la historia del hombre, las grandes obras de la literatura y los conceptos filosóficos fundamentales (Dengo, 1998).

La filosofía de una tradición democrática y una base jurídica o normativa expuesta se ha fijado en las estructuras del sistema educativo y en el estilo de relación en las instituciones educativas como, por ejemplo, los procesos de gobierno estudiantil que se practican en las instituciones escolares y el actual "ensayo" diario del canto del Himno Nacional y el saludo al Pabellón Nacional.

Como se planteó anteriormente, el enfoque de los sistemas simbólicos considera importante el dominio cultural y universal como indispensables, según sea el caso, para enfrentar el ambiente social en que se ven inmersos.

Sin lugar a dudas, los fines de la educación costarricense responden, bajo este enfoque, a una manera de interpretar la formación que tendrá el "aprendiz", argumentada en los objetivos del I y II ciclos de la Educación Primaria; esta "simbología" característica de nuestra sociedad, conlleva a valorar la importancia de cómo se ha distinguido el país a nivel internacional 
por sus principios de convivencia a través del diálogo y la sustentación de este importante hecho con la derogación del ejército nacional a pesar de los conflictos en países vecinos.

Por otro lado, este conocimiento universal plasmado en los fines educativos del contexto costarricense considera una formación integral, o sea, la contribución de un "ciudadano" amante de sus deberes cívicos es su médula espinal, así como estimular un desarrollo de solidaridad y respeto entre los seres humanos para alcanzar la comprensión social.

Goleman (1995) considera que para desempeñarse efectivamente en el mundo social, el niño necesita aprender a reconocer, interpretar y responder en forma apropiada las situaciones sociales. Es decir, la familia o el hogar es vital para la adaptación del sujeto a su medio; esos espacios conforman parte del "hábitat" que favorece la asimilación como una especie, en la biodiversidad de "ecosistemas" y, facilita las condiciones ambientales necesarias, que constituyen un "nicho humano", determinando la ocupación y convivencia del individuo para un estilo de vida en sociedad.

Con el tiempo, crecen las unidades sociales de familias y tribus a comunidades y sociedades más complejas, en dicha expansión se originan nuevos mecanismos de acomodación y se auspicia el surgimiento de otras formas de organización social, que por las tendencias de los sistemas de valores, orientados en las relaciones con el ambiente natural, con otra especies y otros seres humanos. Se designan a numerosas funciones a favor de estos organismos sociales especializados como el caso de la educación, la salud, el mercado que resaltan el vínculo entre el individuo y su medio sociocultural.

El proceso de socialización, como ocurre con todas las capacidades de la inteligencia emocional, comienza con una combinación del temperamento heredado del niño y su reacción ante él. Los niños pequeños difieren en su reacción social y varían considerablemente en su capacidad de respuesta, adaptación y persistencia.

Los adultos, por otra parte, se ven influidos por la conducta de los niños, dedicando más tiempo y atención a los que son más sociables. A menudo los adultos no se dan cuenta de que casi al comienzo de su desarrollo los niños se vuelven socialmente conscientes y sensibles de los matices sociales. La sensibilidad social aparece más regularmente cuando los niños experimentan un número creciente de interacciones satisfactorias con sus pares.

La raíz de la palabra emoción es motio, motions, que se deriva del verbo latino movere. Además contiene el prefijo "e-", que implica "alejamiento", lo que sugiere que en toda emoción hay implícita una tendencia o impulso a actuar, que permite enfrentarnos a la vida cotidiana. Por otra parte, define la palabra emoción como “(...) agitación o perturbación de la mente; sentimiento; pasión, cualquier estado vehemente o agitado" (Goleman, 1999, p. 418).

El término, entonces, señala sentimientos, pensamientos, estados biológicos, psicológicos que caracterizan la acción, que dentro del sistema educativo costarricense mezclan la virtud de un niño y niña amante de su formación en un país cívicamente democrático y, de esta manera, se amplía la herencia cultural, que abarca conocimientos sobre la historia del hombre (añadiría de la humanidad).

Por ejemplo, dentro de los fundamentos de la disciplina de Estudios Sociales, se señala como se conforma una asignatura del plan de estudios de la enseñanza primaria que tiene como propósito potenciar en los niños y las niñas las destrezas, conocimientos y habilidades, como herramientas necesarias para permitir su inserción en la sociedad costarricense.

Los Estudios Sociales resultan primordiales en la sociedad moderna; son relevantes para analizar problemas y su resolución. Pretenden facilitar, enriquecer y clarificar conceptos, valores y actitudes, así como motivar el acceso a los nuevos 
conocimientos y destrezas, para el desenvolvimiento social e individual; vivencias que son imprescindibles en la formación de seres humanos íntegros, capaces de enfrentar y resolver los desafíos de la época actual.

Las disciplinas Geografía, Historia y Educación Cívica han sido integradas como los ejes temáticos de esta asignatura. Además, se nutre de otras disciplinas que tienen en común el estudio de la sociedad, como la Sociología, la Politología, la Economía, la Ecología, la Antropología (Programa de Estudios Sociales para I y II Ciclos, 2003).

En este sentido, la disciplina de la asignatura de Estudios Sociales busca cumplir, mediante diversos objetivos en los ciclos de primaria, su propósito de reforzar los conocimientos preliminares sobre la dimensión espacial y temporal; construir una visión integrada de la persona, la escuela y la comunidad; reconocer la necesidad de participar responsablemente en las actividades que se realizan en la casa, la escuela y la comunidad, ya que intenta al mismo tiempo el cumplimiento de los deberes escolares, reconociendo las experiencias personales, familiares y comunales como manifestaciones de la identidad nacional, valorar las tradiciones y bellezas naturales de nuestro país como parte de nuestra idiosincrasia.

Si se entrelazan estos fines y objetivos, se nota como el partir de la inteligencia espacial de los individuos forma parte de la disciplina de Estudios Sociales, debido a que esta se define como

“(..) la capacidad para percibir con exactitud el mundo visual, para realizar transformaciones y modificaciones a las percepciones iniciales propias, y para recrear aspectos de la experiencia visual propia, incluso en ausencia de estos estímulos físicos apropiados" (Gardner, 1993, p. 216).

La operación fundamental de la inteligencia espacial es la habilidad para percibir una forma o un objeto. Para ello, se toma en cuenta la aptitud para reconocer instancias de un mismo elemento, para transformar o reconocer una transformación de un elemento en otro y, la capacidad para evocar la imagen mental y luego transformarla; así como la capacidad de producir una semejanza gráfica de información espacial, como es la lectura de los mapas y el utilizar la rosa de los vientos.

Estas capacidades tienen distintos usos, como el sentido de orientación en una locación para reconocer objetos y escenas en ambientes originales o alterados. Por otro lado, se utilizan cuando se trabaja con descripciones gráficas: diagramas, mapas y otras para comprender sensiblemente diversas líneas de fuerza que entran o salen de un despliegue visual (capacidad utilizada por distintos artistas plásticos o críticos de arte) y la distinción de parecidos en dos formas aparentemente dispares.

La inteligencia espacial proporciona la capacidad de pensar en tres dimensiones, como lo hacen los marinos, los pilotos, los escultores, los pintores y los arquitectos, permite al individuo percibir imágenes externas e internas, recrearlas, transformarlas o modificarlas, recorrer el espacio o hacer que los objetos lo recorran, producir o decodificar información gráfica.

De igual manera, la inteligencia visual-espacial consiste en la habilidad de pensar y percibir el mundo en imágenes. Se piensa en imágenes tridimensionales y se transforma la experiencia visual a través de la imaginación. La persona con alta inteligencia visual puede transformar temas en imágenes, tal como se expresa en el arte gráfico (Hernández y Redondo, 2002).

Por otro lado, se pueden citar los fundamentos del programa de la asignatura de Matemáticas, que justifican su integración en la sociedad moderna como uno de los pilares con un papel creciente del conocimiento en todas las dimensiones de su desarrollo. Las ciencias y la tecnología se han convertido, especialmente después de la Segunda Guerra Mundial, en dispositivos imprescindibles de los planes de 
progreso económico, político y social de las naciones. Existe un cambio de paradigma que “(...) significa una nueva manera de ver el mundo y ha afectado la forma en que las naciones perciben su desarrollo" (Programa de Matemáticas para I y II Ciclos, 2003).

El programa de esta área justifica una de las implicaciones de este cambio decisivo en que aquellas naciones que no logren entender el significado del conocimiento, en este contexto histórico, estarán condenadas al atraso y a menores niveles de calidad de vida para sus poblaciones.

De una manera consciente, un país no desarrollado tiene que invertir decisivamente en el fortalecimiento de las ciencias, tanto naturales como sociales, en la tecnología y en el ensanchamiento cultural de sus pueblos como recursos indispensables de cualquier estrategia de progreso nacional.

La educación, en todas sus dimensiones, aparece en este contexto, no solo como un medio de avance individual, sino como la llave del progreso colectivo y nacional.

Es decir, se tiene que invertir mucha energía y muchos recursos en la educación científica y tecnológica, sin descuidar la perspectiva integral y humanista, que debe constituir el valor central de partida en el decurso nacional.

Por esta razón, la educación debe estructurarse, como lo sugiere Delors (1996), en su libro "La Educación Encierra un Tesoro", en torno a cuatro aprendizajes fundamentales. En cierto sentido, los pilares del conocimiento de aprender a conocer (adquirir los instrumentos de la comprensión), el aprender a hacer (para poder influir sobre el propio entorno), el aprender a vivir juntos (para participar y cooperar con los demás, en todas las actividades humanas) y el aprender a ser (proceso que recoge elementos de los tres anteriores).

Las matemáticas han ocupado un lugar privilegiado en el devenir del conocimiento humano, tanto como descripción de dimensiones especiales de la realidad, como lenguaje y fundamento de las otras ciencias, o sea, una matematización de las otras ciencias como característica constante del conocimiento moderno.

Para poder decir cual es la operación modular de la inteligencia lógico-matemática, debe mencionarse la capacidad de confrontación con el mundo de los objetos, ya sea en su ordenación, reordenación y evaluación de su cantidad.

Gardner (1993, p. 168) opina que

El individuo se vuelve mas capaz para apreciar las acciones que uno puede efectuar sobre los objetos, las relaciones que se obtienen entre estas acciones, las declaraciones (o proposiciones ) que uno puede hacer respecto de acciones reales o potenciales, y las relaciones entre esos enunciados.

Para el autor, el desarrollo humano en la inteligencia lógico-matemática parte de los postulados de Jean Piaget, psicólogo suizo, en referencia a que desde la infancia el niño y la niña se ven envueltos en un mundo de objetos (chupones, móviles, juguetes, etcétera) y pronto se darán a la tarea de observar cómo dichos objetos se comportan en diferentes circunstancias, por simples conexiones causales, siempre y cuando los objetos continúen al alcance de la vista del infante.

Los expertos en la materia destacan la no existencia de diferencias entre la lógica y la matemática, que la capacidad lógico-matemática no es sino una serie de habilidades que se entrelazan entre sí. Se inician con la observación y manipulación de objetos materiales; posteriormente, se continúa a sistemas más formales cada vez abstractos, cuyas interconexiones son cuestiones de la lógica. Tal como lo menciona Russell (citado por Gardner, 1993), al inicio la matemática y la lógica tenían caminos separados, sin embargo, en los tiempos actuales “(...) la lógica es la juventud de las matemáticas y estas son el estado lógico de la lógica" (p. 74).

Los talentosos en las matemáticas encuentran su atractivo en la materia cuando logran resolver un problema que durante mucho tiempo se ha considerado irresoluble, o bien, en inventar un nuevo 
campo en las matemáticas, descubrir nuevos elementos de las matemáticas, encontrar relación entre campos que anteriormente eran ajenos unos de otros desde el punto de vista de las matemáticas.

No cabe duda, que el estereotipo del joven talentoso en las matemáticas es de un individuo aislado y con dificultades en las relaciones interpersonales, pero al parecer encuentran su autosuficiencia en los niveles altos de concentración que requieren para resolver problemas matemáticos.

Gardner (1995) cita una serie de consejos para el matemático y la resolución de sus problemas, por ejemplo, se aconseja generalizar que partan de un conjunto de objetos dado en un problema a un conjunto mayor que contenga el problema dado; así al especializarse, pasen de un conjunto dado de objetos a uno más pequeño, que esté contenido en uno dado; que indaguen analogías, hallando con ello un problema o situación que conlleve similitudes (y diferencias ) instructivas con relación a lo que se considera, para cuando se encuentran un problema difícil, resolver primero uno más sencillo dentro de uno mayor, que busque una solución al componente más simple y, luego, partir de esa solución tratando de demostrar y averiguar las consecuencias de la suposición.

En este sentido, la matemática se ve relacionada con el mundo de la ciencia, en tanto los científicos la utilizan como instrumento indispensable para elaborar modelos y teorías que puedan describir y explicar el funcionamiento del mundo: de objetos (física y química), seres vivos (biología), seres humanos (ciencias sociales, cognitivas $\mathrm{y}$ conducta).

La diferencia básica entre el matemático y el científico estriba en que el primero está interesado en la exploración de sistemas abstractos en sí mismos y el otro desea explicar la realidad física.

Goleman (1995) señala que “(..) la capacidad de pensamiento" es indispensable para un buen rendimiento en el aspecto lógico matemático y, al mismo tiempo una buena "sintonía o empatía" con esta asignatura.

Es decir, a diferencia de cualquier otra especie de animales, los seres humanos tienen la capacidad de inventar y controlar sus emociones simplemente a través del pensamiento.

El desarrollo de la neocorteza, la parte del cerebro que controla el lenguaje y el pensamiento lógico, permite tener pensamientos sobre nuestros sentimientos y modificarlos.

Por lo tanto, el autor de las inteligencias emocionales comunica que antes de convertirnos en una sociedad industrial, los padres enseñaban a sus hijos a distinguir las plantas que podían comer de las que no, como henar un caballo o como cultivar un campo.

Actualmente, los niños necesitan diferentes capacidades para sobrevivir, los padres deben enseñarles a sus hijos a usar su intelecto, así como las capacidades emocionales y sociales a fin de enfrentar una serie cada vez más compleja de tensiones personales, familiares y sociales.

Goleman (1995) cita que la inteligencia emocional abarca varias dimensiones entre ellas la interpersonal, que incluye aspectos como empatía, responsabilidad social, relaciones interpersonales; la intrapersonal que destaca aspectos como el autoconcepto, la conciencia de emociones, la realización personal, la independencia, la asertividad, la adaptabilidad para lo cual son necesarias objetividad, flexibilidad, solución de problemas, el manejo del estrés, que implican tolerancia y control de impulsos.

Por último, el estado general de ánimo, el optimismo o el negativismo, como dimensiones o elementos necesarios para el desarrollo adecuado de la inteligencia emocional.

Los niños y las niñas son muy susceptibles al trasfondo de la frustración, principalmente cuando en nuestra sociedad se privilegian ciertas habilidades sobre otras. Sin subestimar este último comentario, a nivel estadístico según algunos informes 
son alarmantes los niveles de repitencia y bajo rendimiento académicos en el área de matemáticas. La experiencia como docente me permite postular la tesis de que en muchas ocasiones no preparamos al niño y a la niña a desarrollar un pensamiento lógico y ordenado, por ejemplo, actualmente me dedico a enseñar las tablas de multiplicar a los niños de segundo grado, pero estas no pueden ser comprendidas, asimiladas y acomodadas, como cita Piaget, en su mente de manera simbólica (según el enfoque tratado) por no entender el proceso de conteo por series numéricas, o sea, conteo de dos en dos, tres en tres ..., y la suma sucesiva.

En este sentido, escuché a una colega afirmar que en el proceso de la lógica matemática, una habilidad tan simple como es dominar el concepto de suma, resta facilita o limita al infante para adquirir otras habilidades.

Esto me hace recordar la siguiente experiencia con un grupo, cuando intentaba que asimilaran el concepto de cuerpo geométrico y la diferencia de una figura plana, desarrollé la estrategia de solicitar un anuncio de un jugo, así como llevarles un envase de jugo de diferentes sabores a cada niño y niña. La dinámica fue simple, pero relevante a los ojos de los aprendices y las aprendices; al inicio, ofrecí el jugo a cada uno y empezamos a discutir de manera simple (sin la sensación de que averiguaba para un tipo de evaluación) y formulé preguntas, así al cabo de un momento, introduje la temática intentando que respondieran la diferencia entre el "anuncio" del jugo y el envase que tenía en la mano. Este ejercicio simple brindó el beneficio de que alrededor de un ochenta y cinco por ciento reconociera una figura plana y la diferenciara de un cuerpo geométrico.

De igual manera, puedo ejemplificar otros aspectos que pueden parecer comunes y trillados, aunque al fin y al cabo en mi función como facilitador han dado resultado.

Por ejemplo, en un grado específico cuando trataba la temática de los procesos de la evolución de los seres vivos: nacer, crecer, reproducir y morir; invité a los niños y a las niñas a traer fotografías desde cuando estaban en el vientre de la madre hasta su edad actual para evidenciar las etapas que constantemente vivimos todos los seres vivos; igualmente llevé un vídeo sobre la evolución de una mascota. En la película, se observó con claridad el proceso evolutivo de una planta y de un animal.

Gracias a Dios que la tecnología facilita muchísimo estos procesos que se relacionan claramente para que luego el estudiante pueda interiorizar el concepto de historia y de una idea de la línea temporal a pesar de ser de una asignatura de Ciencias, es factible desarrollar temáticas con otras áreas de Estudios Sociales y Español.

La finalidad de estos ejemplos es clarificar la importancia de la funcionalidad del papel docente en el campo de la formación de un niño y una niña, y el modo de despertar la curiosidad en ellos y en ellas sobre su medio.

\section{La educación costarricense y las inteligencias múltiples en el salón de clase}

Gardner (2000) propone una reforma total al sistema educativo actual, la cual está basada en las teorías viejas de la inteligencia en donde se da énfasis en el currículo a las áreas lógico-matemática y la lingüística. Este gestor de la teoría propone que la educación se centre en el individuo no egocéntricamente, pero sí dándole oportunidad de desarrollar sus diferentes inteligencias.

De esta manera, se busca el desarrollo de todas las áreas y no frustrar a los estudiantes que no tienen las inteligencias mencionadas en las teorías tradicionales. Además, el sistema educativo antiguo servía a las diferentes épocas, ya que un individuo que estudiaba mucho posiblemente dominaba las diferentes áreas del conocimiento. Sin embargo, hoy en día es imposible dominar una sola área debido a la gran cantidad de información disponible. 
Por lo tanto, indica que debe maximizarse la capacidad del individuo en cierta área, por ejemplo, si una persona tiene gran capacidad intrapersonal esta debe estimularse al máximo.

Gardner (2000) señala que si se llega a dar esta reforma educativa, un gran porcentaje de estudiantes lograrán encontrar su vocación y oficio, de modo que se logren mayores resultados positivos para la comunidad. También se reduciría probablemente la frustración de muchos ante el hecho de sentirse "incompetentes" en una estrecha banda de inteligencias específicas.

No obstante, en el presente no hay voluntad de ningún tipo para realizar este cambio educativo que según el autor es necesario y para el cual plantea al menos la integración de cuatro factores (Gardner, 1995).

1. La evaluación: diseñar procedimientos e instrumentos que permitan valorar al estudiante de forma neutra y evaluar, permitiendo observar el tipo de aprendizaje que se desea observar.

2. Currículo: modificar los programas educativos para que se centren en conocimientos necesarios actuales y no en los que fueron utilizados en el pasado, o sea, deben ser comprensibles y adaptarse a los diferentes estilos o capacidades de aprendizaje.

\section{Educación de profesores $\mathbf{y}$} desarrollo profesional: capacitarse en los diferentes tipos de aprendizaje de los estudiantes. Deben de ser más sensibles a las necesidades de aprendizaje de cada individuo. Los nuevos profesores deben formase con estas ideas y deben transmitir ese conocimiento a las nuevas generaciones.

\section{Participación de la comuni-} dad: la sociedad no puede darse el lujo de descargar el proceso de aprendizaje en la instituciones educativas, pues las nuevas necesidades de educar a los diferentes tipos de personas, originan la necesidad de una participación activa de la comunidad. Esto se logra mediante la cooperación de órganos privados y estatales.

Gardner (2000) afirma que lentamente se han hecho modificaciones en el currículo actual para satisfacer la diferentes necesidades educativas de las personas como individuos y no como masas, sin embargo, realmente falta mucho por hacer como se citó al inicio de este artículo.

El docente puede analizar algunas preguntas difíciles sobre factores que promueven o interfieren con el aprendizaje, debido a que a la hora de aplicar las Inteligencias Múltiples (I.M.) es conveniente reestructurar el aula para crear áreas o centros de interés que se dediquen a inteligencias específicas, como medio para ampliar los parámetros para la exploración de cada dominio por los alumnos.

La teoría de las I.M. puede tener un efecto de gran importancia en la forma de actuar de los alumnos en el aula, por la simple razón de que se crea un entorno en el cual se reconocen las necesidades individuales y se busca solucionarlas a lo largo de todo el día escolar. Hay menos probabilidades de que se sientan confundidos, frustrados o tensos en un medio como este y es menos frecuente la necesidad de recurrir a sistemas disciplinarios complicados.

No obstante, en nuestro contexto sí se pueden citar posibles situaciones que no concuerdan plenamente con lo propuesto por Gardner y es el tiempo, la cantidad de alumnos que tiene un educador, el docente no cuenta con los insumos necesarios para enfrentarse al reto de trabajar por inteligencias múltiples, dejando de lado el contexto inmediato, la jerarquización, la gestión educativa, mecanismos específicos de evaluación formativa y sumativa, la estructura organizativa del sistema educativo costarricense que no considera con claridad el manejo de los niños y las niñas con necesidades educativas especiales.

Finalmente, se destaca que no existen los suficientes recursos económicos 
para reestructurar todos los programas educativos y adaptarlos a un currículo centrado en el individuo. Esto implica no solo crear el currículo, sino que habría que capacitar a todos los profesionales de este campo.

\section{Apreciaciones finales}

El enfoque simbólico en el contexto educativo ofrece el idioma indispensable para la comunicación entre los sujetos y su medio, así como el no verlos como objetos. En este sentido, se puede visualizar una serie de primicias para entender la realidad como la perspectiva ecológica que evidentemente permite a los individuos entender más su funcionalidad y priorizar al mismo tiempo cuales aprendizajes delega en las personas.

Por tanto, en las instituciones debe existir una reorientación bajo este enfoque que brinde mayor participación estudiantil y más contacto con el sector productivo y de servicios, así como la permeabilidad ante la realidad social y una actividad educativa originada en los intereses de la comunidad local o inmediata.

Es decir, se establece la relación entre el docente y el educando, facilitándose la comunicación y estimulándose la creatividad, porque se considera a cada persona única y diferente de las demás, pero comprometida con sus iguales y siempre en la búsqueda de soluciones a los problemas de una manera altruista.

Concuerdo con Gardner al considerar que la función social de la educación como medio para crear las condiciones para la armonía de los grupos, buscando al mismo tiempo que se vayan liberando de sus limitaciones para construir una cultura que desarrolle el diálogo y la conciencia colectiva, frente a un espíritu crítico. Esta posición, al mismo tiempo es sustentada por una corriente pedagógica ecléctica y ecológica, en la cual se conceptualiza el currículo de manera participativa social (integrador) y centrado en la persona (cognitiva, confluente, recurrente y personalizado), orientando al ser humano a descubrir la naturaleza del entorno apropiado para su desarrollo potencial, como máxima expresión del orden natural que debe construir sin destruir el entorno físico, natural y social.

No obstante, se busca una armonía colectiva, una igualdad de derechos en relación con los recursos y el desarrollo individual y colectivo. De esta manera, la educación debe promover el desarrollo armónico y equitativo de la humanidad el cual es responsabilidad, como postulan Powell, Maturana y diversos movimientos del progresismo, de la escuela nueva, eclécticos y ecologistas en los últimos años (Carvajal, 1998).

Sin lugar a dudas, la educación deberá, por tanto, centrarse en cada ser humano y al mismo tiempo en los escenarios en que convive promoviendo una persona activa, emancipadora y gestora de su aprendizaje, sobresaliendo el respeto por sí misma como protagonista, apropiándose de la cultura en su medio ambiente humano y natural reflejado en postulaciones fundamentalistas de la sicología gestal o gestálica.

La perspectiva de Gardner y Goleman en la educación enriquece no solo la adquisición de conocimiento en el alumnado, sino también apoya adaptaciones que promueven un equilibrio integral entre el hábitat y la especie. Es decir, faculta al estudiante para "acogerse" dentro de un medio natural y social, donde el papel del educador es relevante, como bien lo dice Joaquín García Monge en un fragmento del Discurso pronunciado en 1917, con motivo de la Tercera Graduación de la Escuela Normal

El educador no es un domador, es un modelador. No es un verdugo, sino un profeta. No es un empleo que gana sueldos para comer, sino un aislado que vela en silencio por el bien de los demás. No es un miserable, es un poderoso; juega con el infinito porque trabaja con el alma. El maestro no compone, demuele y transforma. Demuele principios tóxicos y absurdos, sistemas nocivos y creencias 
falsas. Reforma temperamentos enfermos, organismos raquíticos, instituciones torcidas y construye sociedades sanas, justas y fuertes.

Sin embargo, no puedo dejar de lado las limitaciones de nuestro sistema educativo para la practica de atender una propuesta como la que he tratado. Es difícil, según mi experiencia profesional, atender la diversidad de niños y niñas que constituyen seres únicos que el sistema homogeniza, aunque “(...) la lealtad primordial esté dirigida al bienestar de toda la humanidad" (Sander, 1996; p. 149).

El sistema educativo costarricense no está preparado desde su funcionalidad administrativa para aplicar un marco referencial teórico tan complejo como el expuesto por estos autores. Deseo que se entienda que esto no quiera decir que está "imposibilitado" para hacerlo, sino más bien que debe replantearse ciertos elementos como la diversidad y capacitar al docente para enfrentarla en sus múltiples funciones, lográndose posiblemente con la reflexión de Hanna (citado por Rivas, 2000) “(...) el cambio planificado implica una decisión consciente y positiva para producir una diferencia deseada."

Es decir, el proceso de dar apertura a los aspectos cualitativos y los cambios que emergen en el contexto internacional favorecen la adquisición de un "sujeto" multifacético que logre enfrentar las situaciones que demanda el contexto para tener éxito en las exigencias diarias de nuestra sociedad cada vez más compleja.

No podemos subestimar, que las inteligencias sobrepesan lo lógico o lo lingüístico, más bien debe llevarnos a reflexionar sobre nuestro "analfabetismo" en miles de aspectos, como el contexto emocional, del cual últimamente ha dado paso una "conciencia" ecológica y la diversidad latente desde hace mucho tiempo, aunque hasta el momento se ha minimizado en las propuestas de un currículo oficial.

Quizá mucho de lo dicho en estas páginas resulte demasiado abstracto $\mathrm{y}$ complejo, pero me parece cimiento imprescindible, sin el que sería imposible realizar un análisis profundo de nuestra situación actual, porque las decisiones son el comienzo de algo y, a pesar de los obstáculos, como cosas espantosas que de vez en cuando hacen que apartemos los ojos de la meta, debemos aprender el lenguaje sin palabras para estar preparados a las sorpresas del tiempo.

El cambio es meta y es camino, es plataforma del pensar, es estrategia y es destino, es torbellino que avanza sin cesar (...) El cambio es un proceso a través del cual, el presente se torna recuerdo y el futuro realidad, la ciencia se hace posible (...)" De la Torre, et al. $(1998$, p. 6).

\section{Referencias bibliográficas}

Ander-Egg, E. La planificación educativa: Conceptos, métodos, estrategias y técnicas para educadores. Buenos Aires, Argentina: Magisterio del Río de la Plata. 1996.

Antúnez, P. Proyecto educativo a la programación del aula. Barcelona: Grao. 1995.

Armstrong, T. Las Inteligencias Múltiples en el aula. Buenos Aires, Argentina: Ediciones Manantial. 1999.

Armstrong, T. Inteligencias Múltiples: Cómo descubrirlas y estimularlas en sus hijos. Bogotá, Colombia: Grupo Editorial Norma. 2001.

Bauer, A. y Shea, T. Educación especial: un enfoque ecológico. 2da. ed. Nueva York, E.E.U.U.: Mac Graw Hill. 1999.

Bronfernbrenner, U. "An integrative perspective on children's divorce adjustment”. American psychologist, 36. Pp. 856- 866. 1979. 
Camacho, M. Estructuras de planificación según los postulados de la Teoría de las Múltiples inteligencias. San José: Instituto Educativo Moderno, Concepción de Tres Ríos. 2003.

Capra, F. "La red de los problemas que hay en el mundo". Nueva Conciencia, 22. Pp. 26 -31. 1994

Carr, W. y Kemmis, S. Teoría crítica de la enseñanza. La investigación acción en el profesorado. Barcelona, España: Martínez Roca. 1988.

Carvajal, C. Teorías educativas, concepciones curriculares y corrientes pedagógicas. Mimeografiado, Escuela de Formación Docente, Facultad de Educación, Universidad de Costa Rica. 1998.

Castillo. Enfoques o concepciones curriculares Currículogeneral. Mimeografiado, San José: Escuela de Formación Docente, Universidad de Costa Rica. 2003.

Centro de Innovación Educativa y Fundación Omar Dengo. Herramientas de apoyo en los ambientes de aprendizaje. San José: Fundación Omar Dengo. 2003.

Chaverrí, G. y Navarro, T. Programa para la atención a la diversidad educativa basado en las teorías de inteligencias múltiples e inteligencia emocional. Un modelo de apoyo metodológico al procesos de enseñanza aprendizaje en el aula. Escuela de Sicología, Facultad de Ciencias Sociales, Universidad de Costa Rica. 2002.

Copeland, C. Teaching-learnig behaviors and the demands of the classroom envioronment. En W. Doyle y T. Good (Eds). Focus in Teaching, 1. Pp. 8397. 1982
De Alba, A. Currículo, crisis, mito y perspectiva. México: Universidad Nacional Autónoma de México. 1994.

De la Torre, S., Jiménez, B.; Tejada, J., Carnicero, P., Borell, N. y Medina, J. Cómo innovar en los centros educativos: estudios de casos. Madrid: Escuela Española. 1998.

Delors, J. La educación encierra un tesoro. Madrid, España: Editorial Santillana- UNESCO. 1996.

Dengo, M. Educación costarricense. San José: Universidad Estatal a Distancia. 1998.

Erickson, F. y Schultz, J. When is context? Some issues and methods in the analysis of social competence, pp. 147-160. En John Green y Chareles Wallat (Eds.) Ethnography and languaje in educational settings. Noawood, New Jersey: Editorial Publishing Ablex. 1981.

Freire, P. La Educación como práctica de la libertad. Madrid, España: Siglo XXI. 1998.

García, J. Discurso con motivo de la tercera Graduación de la Escuela Normal. Mimeografiado, División de Educación Básica, Centro de Investigación y Docencia en Educación, Universidad Nacional. 1917.

García, V., Álvarez, S., Guerrero A., Desantes, J., Fernández, O., Losado, I., García, J., Marín, C., Martínez, A., Pérez, R. y Soler, E. Formación de profesores para la educación personalizada. Madrid, España: Rialp. 1996.

Gardner, H. Estructuras de la mente. La teoría de las inteligencias múltiples. Bogotá, Colombia: Fondo de Cultura Económica. 1993/1994. 
Gardner, H. Inteligencias múltiples. La teoría en la práctica. Barcelona: Piados Ibérica. 1995.

Gardner, H. La Educación de la mente y el conocimiento de las disciplinas: lo que todos los estudiantes deberían comprender. Barcelona: Piados Ibérica. 2000.

Gimeno, S. El currículo una reflexión sobre la práctica. 6 a. ed. Madrid: Morata. 1996.

Goleman, D. La inteligencia emocional. Buenos Aires, Argentina: Javier Vergara. 1995/1999.

González, J. La programación del aprendizaje lingüistico escolar. Madrid: Universidad de Oviedo. 2003.

Gore, A. La Tierra en juego. Buenos Aires, Argentina: Javier Vergara. 1993.

Green, J. y Weade, R. Teaching as conversation and the construction of meaning in the classroom. Ponencia presentada en la reunión Anual de la American Educastional Research Association. 1988 abril.

Grundy, S. Producto o praxis del curriculo. 7a. ed. Madrid, España: Morata. 1991.

Hernández, D. y Redondo, M. Las inteligencias múltiples y en la aplicación en el aula. San José, Escuela Ciencias de la Educación, Universidad Estatal a Distancia. 2002.

Jones, V. Classroom management in the United States: Trends and critical issues. En D. P. Tattam (eds). Management of disruptive pupil behavior in chools. Chichester, Inglaterra: Jhon Wiley. 1986.
Klinger, C. y Vadillo, G. Sicología cognitiva: estrategias en la práctica docente. México: Mc Graw Hill Interamericana. 1999.

Kurdek, L. An integrative perspective on children's divorce adjustment. American Psychologist, 36. Pp. 856866. 1981.

León, A. El maestro y los niños: la humanización del aula. San José: Universidad de Costa Rica. 1998.

Moreno. "Competencias comunicativas múltiples inteligencias y alfabetización en la educación”. Revista Interamericana de Nuevas Tecnologías de la Información, 1 (5). Pp. 56-63. (2000, Enero-Marzo).

Nagi, S. Valores Sociales e Instituciones y el lugar que ocupan los niños: una perspectiva ecológica. Ponencia presentada en XVI Congreso Panamericano del Niño, Washington D. C., E.E.U.U. 1984 mayo.

Peralta, V. El currículo en el jardín infantil: análisis crítico. Mimeografiado, Facultad de Educación, Universidad de Costa Rica. 1987.

Pérez, R. El currículo y sus componentes. Barcelona, España: Oikostar. 1994.

Pinnell, G. y Galloway, C. Human development, language on communication: Then and now. Theory into practice, 26. Pp. 353- 357. 1987.

Programa de Estudios Sociales para I y II Ciclos Programa de estudios. San José: Ministerio de Educación Pública. 2003.

Programa de Matemáticas para I y II Ciclos Programa de estudios. San José: Ministerio de Educación Pública. 2003. 
Riegel, K. Toward a dialectical theory of development. Human Devolopment, 18. Pp. 65-79. 1975.

Rivas, M. Innovación educativa: teoría, procesos y estrategias. Madrid: Síntesis. 2000.

Sander, B. Gestión educativa en América Latina. Buenos Aires: Troquel. 1996.

Savater, F. El valor de educar. Barcelona; España: Ariel. 1997.

Sequeira, A. La planificación curricular. Mimeografiado, Facultad de Educación: Universidad de Costa Rica. 2000.
Stainback, S. y Stainback, W. Aulas inclusivas. Madrid: NARCEA. 1999.

Thomas, E. y Marshall, M. Clinical evaluation and coordination of services: an ecological model. Exceptional Children, 44. Pp. 16-22. 1977.

Torres, J. Globalización e interdisciplinariedad: el currículo integrado. Madrid, España: Morata. 1996.

Whithaker, P. Cómo gestionar el cambio en contextos educativos. Madrid: NARCEA. 1998.

Wittrock, M. La investigación de la Enseñanza: I Enfoque teorías y métodos. Barcelona, España: Piados. 1997. 